\title{
Estudo clínico-patológico do pilomatrixoma palpebral: experiência no hospital universitário Prof. Edgard Santos
}

\author{
Clinicopathologic study of eyelid pilomatrixoma: the experience of the \\ "Hospital Universitá rio Prof. Edgard Santos"
}

\author{
Eduardo Ferrari Marback ${ }^{1}$ \\ Camila Cardoso ${ }^{2}$ \\ Livia Maria Nossa Moitinho ${ }^{3}$ \\ Roberto Lorens Marback ${ }^{4}$
}

\begin{tabular}{|c|}
\hline RESUMO \\
\hline $\begin{array}{l}\text { Objetivo: Relatar a experiência com pilomatrixoma palpebral no Hospital } \\
\text { Universitário Profe. Edgard Santos, em período de } 30 \text { anos. Métodos: } \\
\text { Revisão no Registro do Laboratório de Patologia Ocular, de todos os } \\
\text { casos de tumores palpebrais no período de } 1974 \text { a } 2004 \text {. Após a identi- } \\
\text { ficação dos casos de pilomatrixoma, foram coletados dados referentes a } \\
\text { gênero, idade, tempo de sintomatologia, aspecto, localização e suspeita } \\
\text { diagnóstica inicial. As lâminas coradas pela hematoxilina e eosina foram } \\
\text { revisadas. Resultados: Dentre os } 621 \text { casos de tumores palpebrais, } \\
\text { quatro eram de pilomatrixoma ( } 0,64 \%) \text {. Três pacientes eram do sexo } \\
\text { masculino. A idade média foi de } 25 \text { anos, variando de } 8 \text { a } 54 \text {. O tempo de } \\
\text { sintomatologia médio foi de cinco meses, variando de } 2 \text { a } 12 \text {. Em três casos } \\
\text { a lesão localizava-se na pálpebra inferior. Em apenas um caso o diagnós- } \\
\text { tico clínico foi correto. Ao exame anatomopatológico, dois casos apresen- } \\
\text { tavam pseudocápsula. O caso cuja sintomatologia foi mais prolongada } \\
\text { exibia predominância de células do tipo fantasma. Reação tipo corpo } \\
\text { estranho foi vista em todos os casos, calcificação em apenas um. } \\
\text { Conclusões: Trata-se de tumor benigno bastante raro, acometendo } \\
\text { principalmente jovens. Devido a sua raridade e pleomorfismo à apresen- } \\
\text { tação, dificilmente o diagnóstico clínico é correto. }\end{array}$ \\
\hline
\end{tabular}

Descritores: Pilomatrixoma/diagnóstico; Pilomatrixoma/patologia; Neoplasias palpebrais; Diagnóstico diferencial

\footnotetext{
Trabalho realizado no Departamento de Oftalmologia da Faculdade de Medicina da Universidade Federal da Bahia - UFBA - Salvador (BA) - Brasil.

Oftalmologista do Hospital Universitário Prof. Edgard Santos, Faculdade de Medicina da Universidade Federal da Bahia - UFBA - Salvador (BA) - Brasil.

Médica Estagiária do Serviço de Oftalmologia do Hospital Universitário Prof. Edgard Santos, Faculdade de Medicina da UFBA - Salvador (BA) - Brasil.

${ }^{3}$ Médica Colaboradora do Serviço de Oftalmologia do Hospital Universitário Prof. Edgard Santos, Faculdade de Medicina da UFBA - Salvador (BA) - Brasil.

${ }^{4}$ Professor Titular de Oftalmologia. Faculdade de Medicina da UFBA - Salvador (BA) - Brasil.

Endereço para correspondência: Eduardo F. Marback. Rua Rodrigo Argolo, 89 - Apto. 201 - Salvador (BA) CEP 41940-220

E-mail: eduardomarback@uol.com.br

Recebido para publicação em 09.07.2005

Última versão recebida em 07.02.2007 Aprovação em 16.03.2007

Nota Editorial: Depois de concluída a análise do artigo sob sigilo editorial e com a anuência da Dra. Zélia Maria da Silva Correa sobre a divulgação de seu nome como revisora, agradecemos sua participação neste processo.
}

\section{INTRODUÇ̃̃̃O}

O pilomatrixoma ou epitelioma calcificante de Malherbe é um tumor benigno que acomete preferencialmente pacientes jovens e tem origem no folículo piloso. Embora as pálpebras sejam um sítio de predileção do tumor, a prevalência é baixa ${ }^{(1-3)}$. Devido a sua raridade, freqüentemente causa confusão no diagnóstico clínico ${ }^{(4)}$.

O objetivo deste trabalho é relatar nossa experiência com esta lesão em um centro de referência para tumores oculares ao longo de 30 anos.

\section{MÉTODOS}

Foi feito levantamento no Registro do Laboratório de Patologia Ocular do Hospital Universitário Professor Edgard Santos, Universidade Federal da Bahia, entre os anos de 1974 e 2004 para identificar os casos de tumor palpebral e, dentre estes, os de pilomatrixoma. Os prontuários foram revisa- 
dos sendo anotados dados referentes ao gênero, idade, tempo de sintomatologia, aspecto, localização e suspeita diagnóstica inicial. As lâminas coradas pela hematoxilina e eosina, obtidas do material excisado, foram revisadas.

\section{RESULTADOS}

Dentre os 621 tumores palpebrais submetidos a estudo anatomopatológico, foram identificados 4 casos de pilomatrixoma (0,64\%) ocorridos nos anos de 1990, 1992, 2003 e 2004 respectivamente.

Os dados referentes ao sexo, idade, tempo de aparecimento da lesão, localização e a suspeita diagnóstica em cada caso encontram-se no quadro 1.

A idade média ao diagnóstico foi de 25 anos, variando de 8 a 54 (mediana de 19,5 anos). O tempo médio de sintomatologia foi de 5 meses, variando de 3 a 12 (mediana de 3 meses).

Em relação ao aspecto da lesão: caso 1 - apresentava nódulo subcutâneo; caso 2 - tumor séssil de superfície necrótica; caso 3 - tumor pediculado causando ectrópio, com pequenas áreas de aparente ulceração na superfície (Figura 1); caso 4 - nódulo de aspecto cístico.

Todos os casos tiveram acometimento de pálpebras à esquerda. Apenas no caso 3, paciente com oito anos de idade, o diagnóstico de pilomatrixoma foi aventado pré-operatoriamente. Nos outros casos, o diagnóstico de pilomatrixoma foi feito após identificação dos achados característicos ao estudo anatomopatológico.

Nos casos 1, 3 e 4 o padrão anatomopatológico mostrou células dos tipos basalóide e fantasma em proporção equilibrada. O caso 2 exibia predomínio de células tipo fantasma. Em todos os casos havia áreas de reação granulomatosa tipo corpo estranho com células gigantes multinucleadas. Focos de calcificação foram identificados no caso 3 . Os casos 1 e 4 eram delimitados por uma condensação de tecido fibroso formando uma pseudocápsula (Figura 2).

\section{DISCUSSÃO}

A primeira descrição de pilomatrixoma data de $1880^{(5)} . \mathrm{Na}$ literatura oftalmológica, encontramos os primeiros casos relatados em $1951^{(5-6)}$. Desde então algumas pequenas séries e, mais freqüentemente, relatos de casos isolados têm sido encontrados. Para se ter uma idéia da baixa prevalência vale observar que durante os 30 anos de Registro do nosso Laboratório de Patologia Ocular, analisando mais de 6.000 espécimes, 621 dos quais correspondendo a lesões palpebrais, encontramos somente 4 casos de pilomatrixoma, correspondendo a apenas $0,64 \%$ de todas as lesões palpebrais estudadas. Mesmo quando se considera apenas pacientes jovens, nos quais tipicamente a incidência é maior, a lesão é muito rara. Doxanas e colaboradores, estudando 398 casos de tumores palpebrais em menores de 15 anos, coletados em um intervalo de 50 anos, encontraram 5 casos de pilomatrixoma $(1,25 \%)^{(2)}$.
Na nossa série de lesões palpebrais, havia 46 pacientes com menos de 15 anos sendo o pilomatrixoma diagnosticado em dois destes, ou seja 4,34\%.

Como observado neste estudo, a prevalência é maior nas

\begin{tabular}{|lcccccc|}
\hline \multicolumn{6}{|c|}{ Quadro 1. Características dos pacientes } \\
Caso & Sexo & Idade* & Tempo** & Localização & Suspeita \\
1 & $\mathrm{M}$ & 12 & 3 & $\mathrm{PS}$ & Cisto de inclusão \\
2 & $\mathrm{~F}$ & 27 & 12 & $\mathrm{PI}$ & TU a esclarecer \\
3 & $\mathrm{M}$ & 8 & 3 & $\mathrm{PI}$ & Pilomatrixoma \\
4 & $\mathrm{M}$ & 54 & 2 & $\mathrm{PI}$ & Cisto de Moll \\
*em anos; **em meses; PS= pálpebra superior; Pl=pálpebra inferior; TU=tumor \\
\hline
\end{tabular}

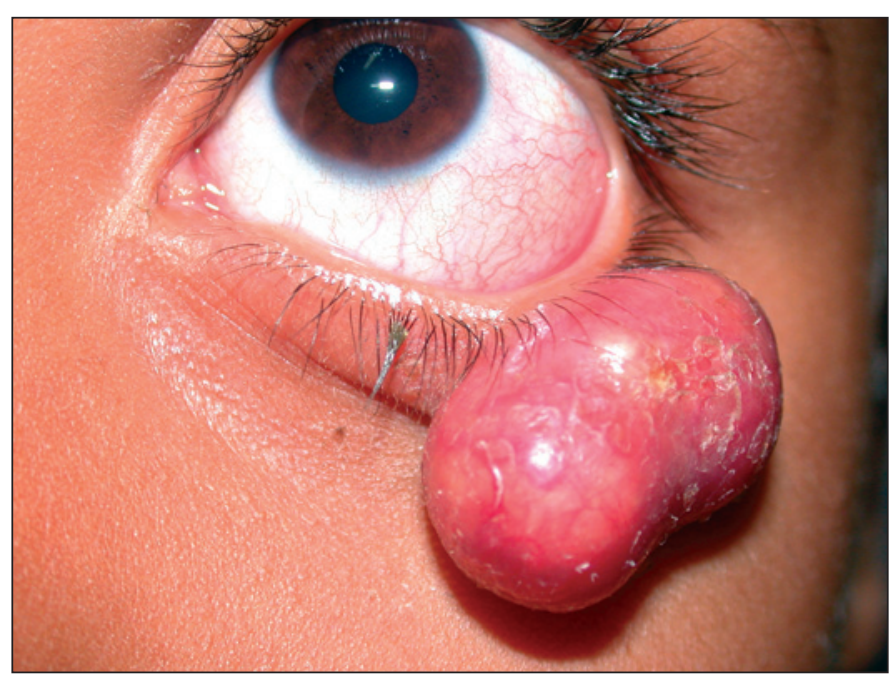

Figura 1 - Caso 3 - Tumor pediculado ocupando a metade externa da pálpebra inferior, com superfície ulcerada e causando ectrópio mecânico

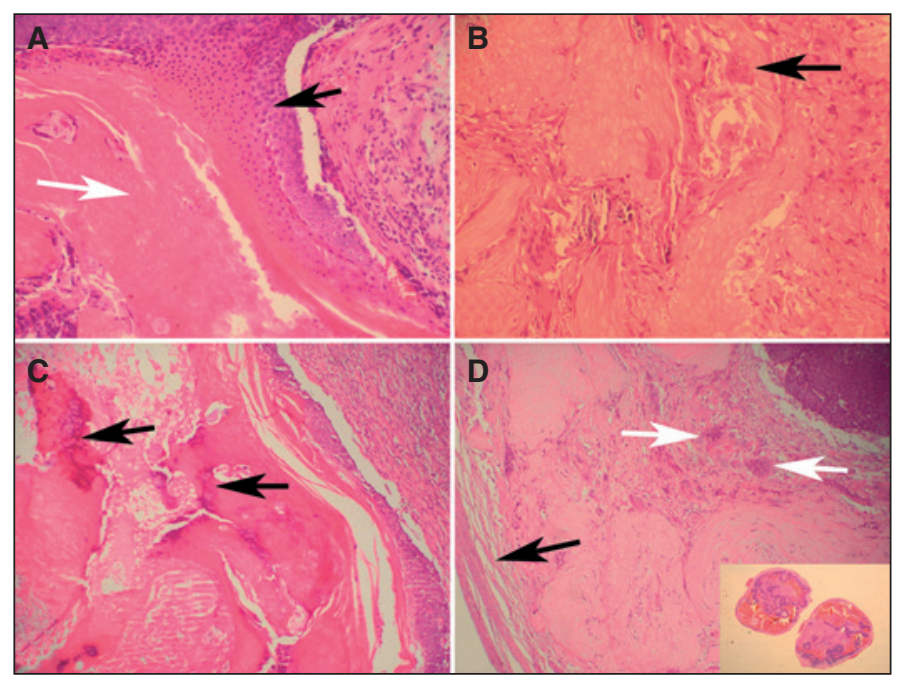

Figura 2 - Aspecto anatomopatológico (hematoxilina-eosina): A) equilíbrio entre células basalóides (seta preta) e células fantasmas no caso 3 (seta branca); B) predomínio de células fantasmas no caso 2 exibindo células gigantes multinucleadas (seta); C) áreas de calcificação no caso 3 (setas); D) pseudocápsula no caso 4 (seta preta e no detalhe) e presença de células gigantes multinucleadas (setas brancas) 
três primeiras décadas de vida, embora possa ocorrer em qualquer idade. Nos 11 casos descritos por alguns autores, todos os pacientes tinham menos de 20 anos e 8 casos até 10 anos, já na série de 67 casos de outros autores, a idade média foi de 26 $\operatorname{anos}^{(4,7)}$.

É sabido que o pilomatrixoma possui algumas características clínicas que podem sugerir o diagnóstico, como a maior incidência em jovens, coloração azulada ou azul avermelhada e crescimento relativamente rápido ${ }^{(4,8)}$. Apesar disso o correto diagnóstico clínico raramente é feito quando o paciente é avaliado $^{(4-5)}$. Nos nossos casos, houve pleomorfismo no aspecto à apresentação que se reflete nas diversas suspeitas clínicas exibidas. Os casos 1 e 4, que apresentavam pseudocápsula ao estudo anatomopatológico, clinicamente exibiam aspecto cístico.

Embora não tenhamos encontrado referência à formação de pseudocápsula na literatura oftalmológica, pilomatrixomas de outras regiões freqüentemente a exibem ${ }^{(9)}$. Dentre os principais erros de diagnóstico relatados na literatura, estão os cistos dermóide e de inclusão, especialmente quando o tumor tem localização subcutânea, como aconteceu com o caso $1^{(4)}$. Outros diagnósticos clínicos um pouco mais fáceis de diferenciar são o hemangioma capilar e o rabdomiossarcoma ${ }^{(10)}$.

Apesar da dificuldade inicial para o diagnóstico clínico, o aspecto à histopatologia é único e habitualmente não deixa dúvidas. O tumor apresenta limites precisos e duas populações celulares predominantes. A primeira é composta por células basofílicas ou basalóides, que lembram células do carcinoma basocelular possuindo núcleo basofílico e citoplasma esparso. O segundo tipo celular é composto por células pálidas e eosinofílicas anucleadas, tal padrão é descrito como de células fantasma. Em tumores mais antigos, as células fantasmas tendem a predominar, como no nosso caso 2. Eventualmente podem ser encontradas áreas de calcificação (caso 3), reação granulomatosa tipo corpo estranho (todos os casos) e até ossificação ${ }^{(1-2,9)}$. Equívoco diagnóstico em biópsia por agulha fina já foi descrito, sendo as células basalóides de um pilomatrixoma localizado sobre a parótida, tomadas como células intermediárias de carcinoma mucoepidermóide ${ }^{(11)}$. Apesar de existir uma variante maligna, o carcinoma pilomatrixial, este é ainda mais raro que o pilomatrixoma e usualmente acomete pacientes idosos ${ }^{(12)}$.

\section{CONCLUSÃO}

Em suma, trata-se de um tumor palpebral benigno que predominantemente acomete pacientes jovens, muito raro, de crescimento relativamente rápido e que dificilmente é clinicamente suspeitado. O tratamento é cirúrgico e o aspecto histopatológico característico.

\section{ABSTRACT}

Purpose: To report the experience with eyelid pilomatrixoma during 30 years in a university hospital. Methods: A review of the Ophthalmic Pathology Laboratory at the "Hospital Universitario Prof. Edgard Santos", was conducted to identify eyelid tumor cases from 1974 to 2004. After identification of pilomatrixoma cases, a chart review retrieved data regarding gender, age, duration of symptoms, gross aspect, anatomic localization and clinical diagnosis. The hematoxilin and eosin stained sections were reviewed. Results: Of a total of 621 eyelid tumors, four $(0.64 \%)$ were pilomatrixomas. Three patients were male. The mean age at diagnosis was 25 years (from 8 to 54). Mean duration of symptoms was 5 months (from 2 to 12). In three cases the lesion was located in the inferior lid. Only one patient had a correct clinical diagnosis. Two cases exhibited a pseudo-capsule on histology. The case with 12 months of history was composed mainly of shadow cells. Foreign body reaction was seen in all cases, calcification in only one. Conclusion: This is a very rare benign tumor of young patients. Due to its rarity and variable clinical presentation, clinical misdiagnosis is common.

Keywords: Pilomatrixoma/diagnosis; Pilomatrixoma/pathology; Eyelid neoplasms/pathology; Diagnosis, differential

\section{REFERÊNCIAS}

1. Boniuk M, Zimmerman LE. Pilomatrixoma (benign calcifying epithelioma) of the eyelid and eyebrow. Arch Ophthalmol. 1963;70:399-406.

2. Doxanas MT, Green WR, Arentsen JJ, Elsas FJ. Lid lesions in childhood: A histopathologic survey at the Wilmer Institute (1923-1974). J Pediatr Ophthalmol. 1976;13(1):7-39.

3. Orlando RG, Rogers GL, Bremer DL. Pilomatrixoma in a pediatric hospital. Arch Ophthalmol. 1983;101(8):1209-10.

4. Yap EY, Hohberger GG, Bartley GB. Pilomatrixoma of the eyelids and eyebrows in children and adolescents. Ophthal Plast Reconstr Surg. 1999;15(3):185-9.

5. Ashton N. Benign calcified epithelioma of the eyelid. Trans Ophthalmol Soc UK. 1951;71:301-7.

6. Malherbe A, Chenantais J. Note sur l'epitheliome calcife des glandes sebacees. Prog Med (Paris). 1880;8:826-8.

7. Ni C, Kimball GP, Craft JL, Wang WJ, Chong CS, Albert DM. Calcifying epithelioma: A clinicopathological analysis of 67 cases with ultrastructural studies of 2 cases. Int Ophthalmol Clin. 1982;22(1):63-86.

8. Shields JA, Shields CL, Eagle RC Jr, Mulvey L. Pilomatrixoma of the eyelid. J Pediatr Ophthalmol Strabismus. 1995;32(4):260-1.

9. Lever WF, Schaumburg-Lever G. Histopathology of the skin. 7th ed. Philadelphia: J.B. Lippincott; 1990. p.578-650.

10. Mathen LC, Olver JM, Cree IA. A large rapidly growing pilomatrixoma on a lower eyelid. Br J Ophthalmol. 2000;84(10):1203-4.

11. Lemos LB, Brauchle RW. Pilomatrixoma: a diagnostic pitfall in fine-needle aspiration biopsies. A review from a small county hospital. Ann Diagn Pathol. 2004;8(3):130-6. Comment in: Ann Diagn Pathol. 2005;9(3):182-3.

12. Hardisson D, Linares MD, Cuevas-Santos J, Contreras F. Pilomatrix carcinoma: a clinicopathologic study of six cases and review of the literature. Am J Dermatopathol. 2001;23(5):394-401. Comment in: Am J Dermatopathol. 2002; 24(5):446; author reply 446-7. 